\title{
Enquêter auprès des chrétiens d'Irak : considérations méthodologiques sur un terrain en «milieu difficile »
}

\section{David Villeneuve}

\section{(2) OpenEdition Journals}

Édition électronique

URL : https://journals.openedition.org/cdg/1638

DOI : $10.4000 /$ cdg. 1638

ISSN : 2107-7266

Éditeur

UMR 245 - CESSMA

Référence électronique

David Villeneuve, «Enquêter auprès des chrétiens d'Irak : considérations méthodologiques sur un terrain en « milieu difficile » », Carnets de géographes [En ligne], 11 | 2018, mis en ligne le 15 septembre 2018, consulté le 21 mai 2021. URL : http://journals.openedition.org/cdg/1638 ; DOI : https://doi.org/ $10.4000 /$ cdg. 1638

Ce document a été généré automatiquement le 21 mai 2021.

\section{cc) (†)}

La revue Carnets de géographes est mise à disposition selon les termes de la Licence Creative Commons Attribution - Pas d'Utilisation Commerciale - Pas de Modification 4.0 International. 


\title{
Enquêter auprès des chrétiens d'Irak : considérations méthodologiques sur un terrain en « milieu difficile»
}

\author{
David Villeneuve
}

\section{Introduction}

1 Certains contextes se prêtent mieux que d'autres à l'investigation scientifique. Or le chercheur doit-il pour autant capituler devant des conditions défavorables à la recherche, qui entravent incontestablement le bon déroulement de son travail ? Et si la solution à ce type de problème venait de la science elle-même?

2 En sciences sociales, l'enquête de terrain est devenue avec le temps une méthode de plus en plus préconisée dans la production des données primaires. Une littérature abondante permet désormais de fixer des paramètres méthodologiques qui garantissent la scientificité d'un processus de recherche ayant recours à cette forme de collecte d'informations. Mais il s'avère que l'environnement général de certains terrains d'enquête rend presque impossible une mise en application rigoureuse des principes de la science, sans que cela ne mette en danger le chercheur qui y opère, ni en question la recherche qu'il y mène.

3 Cet article propose de s'intéresser à la notion de "milieu difficile», tels que le nomment Boumaza et Campana (2007), à travers nos yeux de géographes, en utilisant le cas de la région du Kurdistan irakien, où nous avons mené un travail d'enquête de terrain en 2016 et en 2017 dans le cadre de la préparation d'une thèse de doctorat portant sur les déplacés chrétiens de la plaine de Ninive. Le présent exercice sera l'occasion de réfléchir sur notre méthode, fortement inspirée par l'anthropologie. Dans un premier temps, il sera donc question de situer le travail de recherche en milieu difficile face à l'enquête de terrain en sciences sociales. Dans un deuxième temps, il 
s'agira de décrire en quoi le fait de réaliser un travail de recherche en Irak impose au chercheur des contraintes majeures sur le plan méthodologique. Enfin, nous exposerons certaines des pistes que nous avons préconisées afin de concilier respect des impératifs scientifiques et sécurité du chercheur dans l'accomplissement de son travail.

\section{La recherche en terrain difficile}

4 Selon Mellos (2006), pour qu'il soit question de science, il faut admettre qu'en ellemême la science est une "convention ». Une application stricte de la méthode et des règles de la science est nécessaire afin de respecter la logique de la neutralité de la science. Pour être valable, le processus scientifique requiert trois conditions: l'observabilité, la reproductibilité et l'intersubjectivité.

5 L'observabilité implique qu' " un objet d'analyse scientifique ne peut pas être l'apanage de la seule expérience d'un individu isolé ; il doit être ouvert à l'observation publique " (Mellos, 2006: 544). La reproductibilité figure qu'il faille» [...] être en mesure de reprendre les tests empiriques sur le même objet ou sur des objets similaires appartenant à la même classe. La technique d'observation utilisée doit permettre cette reproduction de la vérification empirique» (2006: 544). Quant à elle, « l'intersubjectivité suppose que toutes les étapes de la méthode puissent être traduites en termes publics de sorte que tous les tests puissent potentiellement être menés par au moins deux scientifiques » (Mellos, $2006: 545$ ).

6 Le mode de production scientifique présenté par Mellos (2006) traduit la conception d'une science moderne basée sur l'empirisme. Or, cette représentation de la science ne serait valable qu'en ce qui concerne les sciences expérimentales (sciences de la nature et sciences physiques). En effet, selon Olivier de Sardan (2004; 2008), anthropologue, qui s'appuie sur Passeron (1990), les modes de production de la connaissance en sciences sociales diffèrent de ceux des sciences expérimentales. Simplement, par exemple, un fait historique ne peut être reproduit afin qu'il puisse à nouveau être observé.

7 Pour Boumaza et Campana (2007), politologues, il n'est plus nécessaire aujourd'hui de justifier, voire de défendre le choix de recourir à l'enquête de terrain comme méthode pour faire de la recherche. Toutes les disciplines en sciences sociales y ont désormais recours (Poupart, 2009). Il peut en revanche s'avérer une lourde tâche de démontrer la qualité méthodologique d'un travail d'enquête de terrain. Pour Olivier de Sardan, « [...] la rigueur de l'enquête de terrain n'est pas chiffrable, à la différence de la rigueur de l'enquête par questionnaire, qui l'est en partie. Il est clair que la validité statistique n'est pas sa spécialité, et qu'elle ne peut être jugée à l'aune de la quantification. Pour autant, la pratique anthropologique n'est pas qu'une simple question de "feeling », elle incorpore et mobilise formation et savoir-faire» (1995: 2). Boumaza et Campana rajoutent : « La méthode ethnographique [...] induit des bricolages méthodologiques qui sont au principe de la transformation de l'objet de la recherche tel qu'envisagé initialement par le chercheur" (2007: 5). Nous l'exposerons à partir de notre expérience dans la troisième partie de notre texte.

Chaque terrain est différent des autres et offre des opportunités qui ne se présentent qu'une seule fois. Un individu peut accepter de se confier une fois, à une personne, et ne plus vouloir le faire par la suite. Des individus peuvent être réunis à un certain 
moment, lors d'une occasion fortuite, et ne plus jamais se revoir. L'espace change et les acteurs qui s'y trouvent aussi. Il faut donc se trouver au bon endroit au bon moment pour être témoin d'un phénomène donné et ainsi pouvoir recenser un fait (Olivier de Sardan, 1995 ; 2004). En Irak, la situation des déplacés chrétiens évolue rapidement. Entre 2015 et 2017, plusieurs familles de déplacés ont quitté le pays pour l'étranger. Certaines personnes rencontrées ne se trouvant plus sur place, il est impossible pour le chercheur de penser s'entretenir avec elles de nouveau, du moins dans leur pays d'origine.

$\mathrm{Au}$ fil des séjours, prolongés ou répétés, le chercheur s'aguerrit dans «l'art » de la recherche sur le terrain. Avec le temps, le chercheur apprend à connaître son terrain. Il en comprend les composantes (humaines comme physiques), leurs interactions, leurs particularités et spécificités, de même que leurs subtilités. Le chercheur apprend à surmonter les obstacles que lui présente son terrain, tout autant qu'il en évite de mieux en mieux les pièges et sait reconnaître plus aisément les avenues à emprunter pour tirer de son terrain les meilleures informations. Pour un chercheur, passer du temps sur son terrain est une nécessité. Il faut avoir commis des erreurs pour ne pas les reproduire. Il faut avoir utilisé certaines techniques d'enquête pour savoir qu'elles ne sont pas efficaces. Il faut avoir parlé aux mauvaises personnes pour savoir à qui parler. Il faut s'être fait mentir pour savoir que l'on se fait dire la vérité. Il faut avoir été maladroit pour être habile. Il faut enfin avoir perdu du temps pour reconnaître que ce « temps perdu » était finalement très riche en informations (Olivier de Sardan, 1995 ; 2004). Par exemple, nous avons constaté en Irak que les déplacés chrétiens ont souvent tendance à vouloir cacher leur intention de quitter le pays. Avec le temps, on en vient à comprendre que les chrétiens irakiens se sentent investis par le devoir d'assurer le maintien de la présence chrétienne en Irak, vieille de près de 2000 ans. Mais dans l'adversité, beaucoup d'entre eux quittent le navire, ce qui n'est pas toujours bien perçu socialement.

Boumaza et Campana (2007) apportent à la méthode de l'enquête de terrain une dimension nouvelle, soit celle du «milieu difficile». Le concept de milieu difficile se décline en plusieurs formes: se trouver sur un terrain en situation de guerre ou de conflit, mener une recherche dans une autre langue, devoir assimiler les us et coutumes d'une culture étrangère, ou enfin se trouver en présence de groupes extrémistes ou d'univers clos totalisants. Le travail d'enquête en milieu difficile entraîne un certain danger pour le chercheur. D'abord, son intégrité physique peut être compromise, mais également ses composantes morale ou psychologique. Euvrer en milieu difficile signifie également être confronté à de l'hostilité, qui se manifeste, entre autres, par une certaine méfiance de la part des enquêtés à l'égard du chercheur. Dans un tel contexte, Boumaza et Campana soulignent que: «Les ethnologues et anthropologues montrent en effet que, dans ces environnements, les canons de la méthode sont mis à mal, s'agissant notamment de la neutralité du chercheur, de l'impeccabilité des techniques d'enquête, et ce, d'autant plus que le chercheur adopte une posture réflexive » $(2007: 7)$.

11 Il ne faudrait toutefois pas penser qu'une recherche menée en milieu difficile s'inscrit en dehors de toute rigueur épistémologique et heuristique. Plusieurs procédés méthodologiques, applicables aux "milieux difficiles», garantissent la valeur des données produites par le chercheur sur le terrain. Pour Olivier de Sardan (1995), il faut connaître au mieux son terrain, préparer soigneusement chacun des séjours qu'on y 
effectue et y consacrer du temps. L'auteur parle en ce sens d'imprégnation. Pour Park ${ }^{1}$, il faut «[...] aborder l'étude de la réalité sociale en se rendant sur le terrain et non se borner à compulser des statistiques ou à compiler le travail d'autres à des fins purement académiques. L'expérience personnelle constitue [...] un préalable à la connaissance " (1995: 8). Dans notre cas, c'est la réalisation, à l'été 2015, d'un séjour humanitaire d'une durée d'un mois qui nous a introduits à la réalité des chrétiens d'Irak. Les séjours successifs, que nous avons réalisés en 2016 et en 2017, d'une durée respective d'un et de deux mois, nous ont permis, dans un premier temps, de pousser plus loin notre connaissance du territoire, puis dans un deuxième temps, de réaliser un travail de collection de données.

Cette « habileté » du chercheur, dont parle Olivier de Sardan, résulte de sa capacité à comprendre son terrain dans toutes ses subtilités, auxquelles doivent être attentifs les géographes. En effet, pour Claval (2002), toute problématique en géographie culturelle (humaine) doit tenir compte de trois conditions que sont la matérialité, l'historicité et la géographicité. Cela revient à appliquer la logique de la contextualisation promulguée par l'approche anthropologique, qui stipule qu'aucun fait n'est autonome du contexte général dans lequel il s'inscrit. Cette démarche prend trois dimensions : historique, car elle tient compte de tout événement antérieur permettant de comprendre le présent ; empirique, puisqu'elle renvoie les faits et pratiques isolés aux usages sociaux plus généraux; et systémique, car elle s'intéresse aux phénomènes socioculturels (économique, politique, etc.) liés de près ou de loin à l'objet étudié (Obadia, 2007).

L'enquête de terrain nécessite de la part du chercheur une grande adaptabilité. Tel que le relate Olivier de Sardan (1995), les participants à la recherche ne peuvent pas être choisis à l'avance. Le choix des interlocuteurs s'opère ainsi pour une bonne part par «buissonnement » ou " arborescence ». Par exemple, en 2017, sur recommandation d'un prêtre, nous avons rencontré un autre dignitaire religieux, qui nous a mis à son tour en contact avec les chefs des villages dans lesquels il célèbre des offices. Cela nous a permis de visiter des communautés isolées dont nous ignorions l'existence, ce qui représente un apport fort intéressant à notre recherche.

L'enquête de terrain doit composer avec les intérêts et les motivations des enquêtés. Comme le mentionne Grawitz (2001), l'interviewé peut avoir avantage à s'exprimer ou à taire certaines informations. En souhaitant protéger ses intérêts à travers son discours, l'enquêté peut altérer le travail du chercheur en lui fournissant une information parsemée d'élisions ou de mensonges, ou encore en accordant une importance exagérée aux faits qui vont dans le même sens que ce qu'il veut défendre.

Au sein d'un groupe, il est souvent possible de retrouver une logique récurrente parmi les discours. On parlera alors d'un groupe stratégique, c'est-à-dire un collectif dont les membres perçoivent et se positionnent de manière similaire face à un phénomène donné. Or, les groupes stratégiques ne sont pas fixes. Ils ne concernent pas forcément les mêmes acteurs selon que les problématiques changent. En procédant à la triangulation des données récoltées (croisement opéré entre les informations), le chercheur est à même de discerner les discours discordants, ce qui lui permet d'identifier à quel degré la position dominante au sein d'un groupe est l'affaire de tous (Olivier de Sardan, 2008). Le même auteur évoque également l'étape de la saturation, c'est-à-dire ce stade auquel parvient le chercheur dans l'accomplissement de sa recherche, où plus aucune donnée nouvelle ne lui est rapportée. 
Dans un contexte défavorable à la recherche, comme celui d'un « milieu difficile », estil possible de penser que les embuches auxquelles le chercheur se butte dans la réalisation de son travail lui offrent encore davantage de fil à retordre? En situation de conflit, la perspective des enquêtés, leurs intérêts et les relations qu'ils entretiennent entre eux ne peuvent-ils pas être altérés par des impératifs qui les dépassent? Dans une telle situation, les intérêts défendus par les enquêtés ne sont-ils pas initiés par des motivations autres que celles qui les animent habituellement ? En " milieu difficile ", le rapport entre les acteurs est-il affecté, inversé, amplifié par des conditions qui bouleversent parfois l'ordre et les conventions établis au sein d'un groupe ? Enfin, est-il possible de penser que le stade de saturation ne puisse pas être atteint? Cela pourrait se justifier par le fait que le nombre de personnes nécessaires afin de constituer un échantillon valide ne peut être obtenu, ou encore parce qu'une préoccupation domine au sein d'un groupe sur toutes les autres, ce qui pourrait laisser croire au chercheur que plus rien n'est à trouver, sans que cela ne soit cependant le cas.

\section{L'Irak : un terrain difficile}

17 La République d'Irak est un pays du Proche-Orient ayant pour voisins la Turquie au Nord, l'Iran à l'Est, le Koweït et l'Arabie Saoudite au Sud, la Syrie et la Jordanie à l'Ouest. La population du pays, qui s'élevait en 2014 à 31 millions d'habitants, est pluriethnique et plurireligieuse. Les Arabes constituent la majorité de la population ( $85 \%)$, suivis des Kurdes (10\%) et d'un nombre impressionnant de minorités ${ }^{2}(5 \%)$, parmi lesquelles il est possible de citer les Turkmènes, les Assyriens, les Arabes des marais ${ }^{3}$, les Mandéens, les Sabéens, les Yézidis, les Circassiens, ou les Shabaks. L'islam domine nettement le profil religieux de la société irakienne, puisque $97 \%$ de la population du pays se revendique de cette religion. Les musulmans irakiens sont d'obédience chiite à environ $65 \%$ et sunnite à $32 \%$. Les non musulmans en Irak, environ $3 \%$ des habitants du pays, sont principalement chrétiens, mais également yézidis ou mandéens (Benraad, $2012 ; 2015)$.

18 Politiquement, l'histoire irakienne des cinquante dernières années se profile en deux temps : la période durant laquelle Saddam Hussein détenait le pouvoir, entre 1979 et 2003, et celle ayant suivi sa chute, à compter de 2003, suite à l'invasion américaine. Suite à l'arrivée au pouvoir de Saddam Hussein, de nombreuses avancées ont été enregistrées, parmi lesquelles une hausse du niveau de vie des Irakiens et la mise en place d'un statut de la femme à l'avant-garde en comparaison avec plusieurs autres pays arabes. En contrepartie, le Président irakien a entraîné son peuple dans différents conflits : la guerre contre l'Iran, entre 1980 et 1988, et l'invasion du Koweït (Première Guerre du Golfe), en 1990-1991. La période de Saddam Hussein rime également avec l'embargo imposé à l'Irak par les États-Unis, qui a ruiné le pays et largement appauvri la population irakienne. À la fin de son règne, le raïs irakien a opérée une véritable dérive autoritaire, marquée par une sévère répression à l'endroit des Kurdes et un retour en force de l'islam sur la scène publique, ce qui a entrainé un net recul de la condition des femmes et des droits des minorités religieuses (Sleiman, 2006 ; Benraad, 2015).

19 Suite au départ de Saddam Hussein, l'Irak a rapidement sombré dans une spirale de violence. Infiltré par différentes organisations djihadistes, ce qui était au départ un soulèvement général contre l'occupation américaine a dégénéré en un conflit civil aux 
airs d'affrontement intercommunautaire et interconfessionnel, nourri côté sunnite par le ressentiment à l'endroit du gouvernement pro-chiite de Nourri Al-Maliki, en poste de 2006 à 2014 (Benraad, 2015). Les chrétiens des grandes villes du pays ont très tôt été pris pour cible par différents groupes extrémistes, tant chiites que sunnites (Maisterra, 2015). L'absence de groupes de défense chez les chrétiens en ont fait une proie facile. Dans leur ouvrage respectif, Yacoub (2003), De Courtois (2009) et Guitton (2011) décrivent en détails les atrocités dont les chrétiens irakiens ont été victimes: invectives, menaces de mort, exactions, spoliations, enlèvements, demandes de rançon, sommations à partir, meurtres.

Avant l'invasion américaine de 2003, les chrétiens irakiens, dont la présence remonte à près de 2000 ans, étaient au nombre approximatif de 1,3 million de personnes. Selon ce qu'affirment les dignitaires religieux locaux, ils ne seraient guère plus que 400000 à l'heure actuelle. En à peine plus d'une décennie, la communauté chrétienne d'Irak aurait donc perdu les deux tiers de ses effectifs. La diminution est encore plus marquée en proportion pour cette même période dans la capitale irakienne, Bagdad, où le contingent de chrétiens est passé de 600000 à moins de 100000 âmes. La prise à l'été 2014 par l'Organisation de l'État islamique (EI) de la région de la plaine de Ninive, berceau du christianisme irakien, et dernier refuge en Irak pour les chrétiens, a eu pour effet d'accroître le nombre de départs pour l'étranger. Mais surtout, ce sont 150000 personnes qui ont dû partir à la hâte en pleine nuit afin de trouver asile au Kurdistan irakien. La ville d'Ankawa, banlieue chrétienne d'Erbil, capitale de la région autonome $\mathrm{du}$ Kurdistan irakien, a vu sa population quadrupler en quelques heures dans la nuit du 6 août 2014. À la périphérie de la ville, de nombreux camps de déplacés se sont constitués : Al Amal, Al Karma, Ashti I, Ashti II, Karemless, pour ne nommer que ceuxlà. Des camps abritant des Yézédis ont également été organisés (Sako, 2015 ; Roussel, 2015).

En soi, l'agglomération d'Ankawa, tout comme les régions du Kurdistan irakien éloignées de la ligne de front avec l'EI, ne constituent pas des zones de combat. Malgré un calme relatif, qui laisse croire de facto à un minimum de sécurité garanti, il n'en demeure pas moins que le fait d'enquêter au Kurdistan irakien consiste en un processus parsemé d'entraves majeures sur les plans linguistique et culturel, comportant de surcroît des risques liés à la sécurité. Erbil, lieu d'un attentat à la voiture piégée en 2014, ne se trouvait qu'à une trentaine de kilomètres des pans de territoire contrôlés par l'EI lorsque nous nous y sommes rendus en 2016. Le Kurdistan irakien est également le terrain d'une rivalité politique entre les partisans de l'ex-Président ${ }^{4}$, Massoud Barzani, et ceux de son opposant Jalal Talabani. La question de l'indépendance du Kurdistan irakien fait de plus l'objet d'un vif contentieux avec le gouvernement central de Bagdad. Dans un tel contexte, le fait d'enquêter auprès des déplacés chrétiens attise la suspicion des autorités kurdes. À leurs yeux, les chrétiens demeurent des étrangers, proches de Bagdad, ayant autrefois soutenu Saddam Hussein ${ }^{5}$. Enfin, la société kurde irakienne abrite un certain nombre de sympathisants de l'idéologie salafiste, hostiles aux minorités religieuses.

À partir de 2003, les chrétiens irakiens fuyant les grandes villes du pays (principalement Bagdad, Bassorah, Mossoul et Kirkouk) ont afflué vers le Kurdistan d'Irak. À l'été 2014, c'est la population entière de la plaine de Ninive qui est venue à son tour trouver refuge chez sa voisine kurde. Outre Ankawa, qui concentre la grande majorité des déplacés, les chrétiens de la plaine de Ninive se sont aussi rendus dans les 
villes kurdes de Duhok, Zakho et Souleymania, de même que dans certains villages chrétiens à l'abri des hostilités tels Alqosh et Manguesh. Depuis leur fuite forcée, les chrétiens de la plaine de Ninive, objet de notre enquête, forment un vaste ensemble de déplacés au sein duquel les familles ont souvent été séparées, et dont l'organisation communautaire d'autrefois (politique, économique, religieuse, etc.) n'est plus applicable. S'il est certes possible d'avoir accès aux déplacés pour s'entretenir avec eux, leur vie en contexte de déplacement n'a rien à voir avec celle qu'ils menaient auparavant. Avec la reprise aux mains de l'EI des villages chrétiens de la plaine de Ninive par l'armée irakienne au début de l'année 2017, beaucoup de déplacés chrétiens originaire de la plaine de Ninive ont cru pouvoir rentrer chez eux. Pour plusieurs, l'espoir s'est rapidement dissipé dès que l'ampleur de la destruction des villages de la région a été constatée: maisons incendiées ou tout simplement détruites, églises saccagées, cimetières rasés, monastères explosés.

Parmi les déplacés chrétiens de la plaine de Ninive, la méfiance est souvent de mise à l'égard des étrangers depuis les événements des dernières années. Cela tranche avec l'accueil mémorable normalement réservé aux étrangers comme en témoigne De Charentenay (2008). Depuis 2014, les chrétiens ont l'impression d'être oubliés, de ne pas être considérés dans la planification de "l'après EI » qui se trame en coulisse entre les pouvoirs kurde, irakien et étrangers. Quant à elles, les différentes Églises locales (Église chaldéenne, Église syriaque catholique, Église syriaque orthodoxe, Église apostolique assyrienne de l'Orient) peinent dans une telle situation à assurer pleinement leurs rôles de pilier communautaire et de défenderesses des intérêts des chrétiens auprès des autorités. C'est que leurs moyens structurels, financiers, de même qu'en effectifs humains ont considérablement été réduits depuis 2003. Par exemple, un prêtre rencontré au Kurdistan à l'été 2017 nous confiait qu'ils n'étaient plus que trois prêtres dans le diocèse où il sert, alors qu'une dizaine d'entre eux serait nécessaire pour remplir adéquatement le mandat qu'il leur revient. Moins il y a de familles, moins il y a de candidats à la prêtrise. Il en résulte un affaiblissement de la communauté chrétienne en général. Pour nous, il est donc plus complexe d'envisager passer par le canal d'information traditionnel que constitue le clergé.

\section{Déplacés chrétiens au Kurdistan irakien : quelle stratégie adopter?}

24 La question de la disparition des chrétiens d'Irak est soulevée depuis un certain temps dans la littérature. Cette idée d'une fin possible de la présence chrétienne dans le pays se fonde sur le mouvement d'exode de masse que connaît la communauté chrétienne d'Irak. Le travail d'enquête de terrain que nous avons réalisé en Irak en 2016 et en 2017 avait pour objectif de dresser un portrait de la situation des déplacés chrétiens de la plaine de Ninive, afin d'établir un constat sur les perspectives de cette communauté en Irak. Nous avons ainsi questionné nos interlocuteurs au sujet de leur vision de l'avenir et de leurs intentions pour le futur (demeurer en Irak ou quitter le pays).

Lors de nos séjours d'enquête de terrain, nous avons collaboré avec l'organisation nongouvernementale française SOS Chrétiens d'Orient. Nous reviendrons plus loin sur les avantages et les inconvénients de ce type de collaboration dans le cadre d'une démarche scientifique. Initiée en 2014 suite à la fuite des populations de la plaine de Ninive vers le Kurdistan irakien, l'action de SoS Chrétiens d'Orient en Irak avait à l'origine un double objectif : d'abord, apporter une aide humanitaire d'urgence aux 
déplacés chrétiens, puis conforter les populations éprouvées en leur témoignant le soutien de leurs coreligionnaires de France. Les motivations de l'organisation SOS Chrétiens d'Orient ont un certain temps été remises en question en raison de l'affiliation politique de certains de ses fondateurs avec l'extrême-droite. Les concernés ont répondu à plusieurs tribunes que leurs implications personnelles n'avaient aucun lien avec l'association qu'ils ont créée, qui est apolitique (Houdaille, 2017). Le débat autour de cette question semble être clos aujourd'hui et l'action de l'association est largement reconnue sur le terrain par les acteurs locaux que nous avons rencontrées.

L'intervention en Irak de SOS Chrétiens d'Orient devait à l'origine être temporaire. Son action a cependant duré, en raison du besoin toujours exprimé sur place. Avec le temps, l'association a élargi son mandat. En plus de l'aide d'urgence qu'elle procure encore, SOS Chrétiens d'Orient a participé à la construction d'écoles, de centre communautaires, ainsi que d'autres infrastructures améliorant le quotidien des déplacés. L'aide apportée par SOS Chrétiens d'Orient s'adressait initialement aux seules populations chrétiennes déplacées dans la région du Kurdistan irakien. Désormais, ses activités rejoignent également les Yézidis, et se sont étendues ailleurs en Irak avec la mise sur pied d'une école multiconfessionnelle (chrétiens et musulmans) à Bagdad et d'une clinique fournissant de l'aide d'urgence à Bassorah. Depuis l'été 2017, SOS Chrétiens d'Orient soutient les instances en charge du retour des populations de la plaine de Ninive dans leurs communautés d'origine. Dans son action, l'association collabore donc de près avec les Églises locales, afin d'appuyer les membres de la communauté chrétienne qui le désirent à demeurer en Irak.

Plusieurs organisations humanitaires étrangères assurent une présence auprès des déplacés de la plaine de Ninive. Outre des agences bien connues comme Unicef, USAid, la Coopération française, la Coopération allemande ou Caritas, il est possible de croiser sur le terrain des coopérants de plusieurs associations de la société civile, totalement indépendantes ou associées de près ou de loin à une Église : Aide à l'Église en Détresse, l'œeuvre d'Orient, Fraternité en Irak, Christian Humanitarian Aid, Ninive Horizon. De l'aide, financière, parvient finalement aux déplacés via la diaspora irakienne, mais également grâce à des initiatives comme celles du gouvernement hongrois, qui a promis au printemps 2017 de contribuer à la reconstruction du village de Teleskof, ou encore des Chevaliers de Colomb, qui ont manifesté leur souhait d'appuyer la restauration du village de Karemless. Bien qu'ils puissent compter sur une aide extérieure sans doute plus substantielle que celle dont bénéficient d'autres communautés, les chrétiens d'Irak demeurent néanmoins dans une situation très précaire. Il en va de même en ce qui concerne leur avenir. Isolés, affaiblis et souvent traumatisés, beaucoup parmi ceux qui se trouvent toujours sur place n'entrevoient aucun futur pour les membres de leur communauté en Irak. Pour de nombreux déplacés, la « fin » de l'EI n'est qu'un intermède. Tel que plusieurs l'affirment, « autre chose viendra ». "Les chrétiens ne seront jamais plus tranquilles en Irak ${ }^{6}$. Selon ce que nous a exposé un responsable de SOS Chrétiens d'Orient rencontré en 2016, il s'agit donc également pour son association de redonner confiance aux chrétiens d'Irak.

Parmi l'ensemble des organisations non-gouvernementales venant en aide aux déplacés de la plaine de Ninive, sos Chrétiens d'Orient est la seule à assurer une permanence sur le terrain. De ce fait, elle possède une bonne connaissance de la situation en temps réel et des dangers du terrain. L'association dispose également de moyens logistiques et d'un personnel local. Mais c'est surtout en raison du fait que SOS Chrétiens d'Orient 
souhaite encourager la recherche scientifique portant sur les chrétiens irakiens que l'opportunité d'une collaboration devenait pour nous intéressante. Dans un premier temps, cela permettait d'assurer que notre travail de terrain se déroule dans de bonnes conditions de sécurité, de bénéficier de l'expérience acquise par l'organisation, d'accéder dans un contexte de confiance à des responsables locaux, et de recourir aux interprètes embauchés par l'association afin de pallier notre connaissance limitée de l'arabe et notre méconnaissance du syriaque. Dans un deuxième temps, dans une perspective épistémologique, nous avons pu procéder plus aisément à l'étape de l'imprégnation en limitant grandement les risques. En effet, en ayant pu accéder au réseau de contacts de SOS Chrétiens d'Orient sur le terrain, il nous a été possible d'en apprendre sur les réalités de notre terrain, les acteurs qui y évoluent, les rapports de force exercés, le mode de fonctionnement local, les us et coutumes des différentes populations, etc.

En se référant à Althabe et Hernandez (2004), le chercheur peut occuper, aux yeux des enquêtés, différentes positions, voire jouer certains rôles. Il peut en effet susciter la méfiance lorsqu'il est vu comme étant extérieur à la communauté étudiée ; il peut être sollicité afin d'agir en tant qu'arbitre en situation de conflit, car il est perçu comme détenant la raison; ou encore, il peut être associé à une certaine forme d'autorité, souvent transmise par une autorité reconnue au sein du groupe d'enquêtés. Sur ce dernier point, deux options doivent être considérées : soit certains sujets de l'enquête acceptent de témoigner en raison de la confiance qu'ils portent à l'autorité qui les a encouragés à le faire; soit certains enquêtés peuvent se sentir obligés de corroborer une version "officielle" pour ne pas déplaire, ce qui rejoint les propos de Grawitz (2001).

De plus, le chercheur doit s'assurer de procéder à une objectivation des discours qu'il recueille (Breaud, 1996), c'est-à-dire qu'il doit être attentif au fait que ce qu'on lui rapporte peut camoufler certaines "demi-vérités ", ou encore servir de plaidoyer pour défendre une cause, tenter de convaincre le chercheur, ou chercher à obtenir une garantie en retour (Olivier de Sardan, 1995). Nous estimons qu'il faut également se méfier des discours présentant une réalité altérée visant à lancer un appel au secours. En situation de précarité, tout recours peut servir afin de diffuser un message, ce qui laisse planer le risque que le chercheur soit perçu comme courroie de transmission potentielle.

31 Dans le contexte de notre recherche, toutes ces éventualités doivent obligatoirement être prises en compte. Cela nous a même amenés, au moins en partie, à revoir l'approche méthodologique de notre travail de terrain pour l'année 2017, même si ce que nous avions réalisé en 2016 s'était avéré fructueux. En effet, se pouvait-il que notre "association" avec SOS Chrétiens d'Orient ait été à l'origine de biais dans les témoignages que nous avons recueillis? Nous avions remarqué, en 2016, une nette tendance chez les déplacés que nous avons entendus à implorer que notre travail serve à attirer l'attention de l'opinion internationale sur leur sort. Or, bien qu'elles partageaient les mêmes caractéristiques et une réalité semblable, les personnes que nous avons rencontrées en 2017 étaient davantage portées à nous livrer leur histoire, sans qu'il ne soit question de ce que nous pourrions leur apporter. Nous étions alors uniquement identifiés en tant que chercheurs, ce qui pour nous explique le fait que nous n'ayons pas fait l'objet de requête. 

d'enquête de terrain en 2016 sans le support que nous a apporté SOS Chrétiens d'Orient. De l'autre côté, le fait de gagner en autonomie lors de notre séjour de 2017 nous a ouvert des portes que nous n'aurions pas pu franchir en étant identifiés à une ONG. Par exemple, dans les environs du village d'Alqosh, nous avons rencontré des déplacés originaires de Bagdad qui n'ont jamais été visités par aucune ONG. Nous avons tiré de cette occasion de riches informations sur les déplacés ayant un parcours différent de ce qui est plus habituel d'entendre. Aussi, suite à la rencontre d'un jeune commerçant du même village, nous avons été invités à passer la soirée avec un groupe d'amis. Entre les blagues, nous avons longuement discuté sans tabou au sujet de la situation en Irak et des craintes, comme des attentes qui habitent les jeunes Irakiens. d'Alqosh, aurait-il été possible pour nous de s'y rendre sans que les responsables de SOS Chrétiens d'Orient ne nous accompagnent? Et si ces derniers avaient été avec nous au cours de la soirée que nous avons passée avec des jeunes du village, nous aurait-on parlé aussi ouvertement? Ces deux questions permettent de comprendre le dilemme dans lequel se trouve le chercheur qui évolue en «milieu difficile». En effet, nous estimons qu'il faut parfois accepter de "s'en remettre à quelqu'un", comme nous l'avons fait avec SoS Chrétiens d'Orient, lorsqu'il est impossible pour le chercheur de réunir à lui-seul les paramètres nécessaires à sa recherche en raison des conditions défavorables que présente son terrain. Par exemple, en 2017, nous avons visité avec les membres de SoS Chrétiens d'Orient les villages repris à l'EI par l'armée irakienne de Karemless, de Bakhdida (Qaraqosh en kurde), de Bashiqa et Barzani et de Telefkof. Sans le justificatif de déplacement que nous a offert l'organisation, nous n'aurions sans doute même pas pu passer le premier point de contrôle vers l'ancienne zone de guerre en partance d'Erbil. En contrepartie, selon nous, le chercheur qui œuvre en « milieu difficile» doit savoir reconnaitre lorsqu'il est le temps de faire cavalier seul. Cela requiert bien entendu un certain temps, car le calcul des risques est plus complexe.

Tel que le démontre notre exemple, le chercheur peut parfois se retrouver en position de «dépendance " face à un partenaire s'il veut être en mesure de mener à bien une partie de sa recherche. Cela implique que le chercheur doit demeurer sensible à la mission de son collaborateur et lui signifier de la reconnaissance. Mais en même temps, le chercheur doit garder en ligne de mire l'atteinte de ses propres objectifs et tenter de demeurer neutre. Pour éviter les biais qu'une telle situation peut occasionner, il est ainsi nécessaire de redoubler de vigilance et de s'assurer d'effectuer son travail avec rigueur. Le travail de réflexion " post-terrain ", qui permet de mettre en perspective les propos recueillis, devient ainsi une étape majeure à laquelle il faut accorder la plus grande attention.

35 Les milieux difficiles recèlent des secrets et des logiques qu'il est souvent très compliqué de percer. Dans sa compréhension générale du terrain, le chercheur doit ainsi s'accommoder de certaines réponses incomplètes, voire même accepter de ne tout simplement pas avoir de réponse à ses questions. Nous supposons que cela ne témoigne pas nécessairement d'une mauvaise foi de la part des personnes que l'on interroge, mais qu'il s'agit parfois pour eux de protéger par leur silence certains acquis et d'éviter toute nouvelle forme d'ingérence.

Dans les régions en conflit, les acteurs impliqués sont déjà nombreux. En limitant les informations qu'ils fournissent au sujet du jeu qu'entretiennent ces acteurs dans le 
concret, les enquêtés procèdent, selon ce que nous en comprenons, à une segmentation des relations qu'ils entretiennent. Cela ne signifie pas que le chercheur soit pour autant perçu négativement. On se contente de lui fournir que les informations qui l'intéressent. Cela représente évidemment un défi de plus pour le chercheur évoluant en «milieu difficile », qui doit parfois trouver certaines réponses par lui-même, c'est-àdire en procédant différemment, par observation par exemple, ou encore en variant le plus possible les sources d'information auxquelles il peut avoir accès. Cela peut passer par le fait de changer de partenaire sur le terrain si cela s'avère réalisable. Par exemple, il serait très intéressant pour nous de retourner en Irak et de nous «associer » à une autre organisation, humanitaire ou pas, afin de découvrir d'autres façons de faire et de connaître de nouveaux interlocuteurs.

\section{Conclusion}

37 L'enquête de terrain en « milieu difficile » demande de la part du chercheur une grande adaptabilité. Il faut procéder autrement qu'à l'habitude, car le contexte est différent, ne serait-ce qu'en raison du fait que le chercheur doit en premier lieu s'assurer de garantir sa propre sécurité.

S'il est nécessaire de bien connaître son terrain avant de s'y rendre, sans doute la préparation à l'enquête de terrain en "milieu difficile » nécessite davantage de temps encore. De plus, le contexte général étant en constante évolution, en situation de conflit par exemple, il peut s'avérer ardu pour le chercheur d'établir le calendrier du déroulement de sa recherche. Dès le départ, le chercheur doit être conscient que le fait de pouvoir se rendre sur un tel terrain, une fois, est déjà une chance. En effet, il se peut qu'un chercheur ne puisse plus reconduire un autre projet sur le même terrain. Il lui faudra alors réorienter sa recherche, ou trouver un moyen de procéder autrement. Cela signifie qu'un travail de terrain réalisé en milieu difficile peut s'avérer unique, impossible à reproduire. Il revêt ainsi un grand intérêt pour la science, car il apporte des connaissances qui demeureraient autrement ignorées.

Suite à la tenue d'un référendum sur l'indépendance par les autorités de la région autonome du Kurdistan irakien, le 25 septembre 2017, le gouvernement central de Bagdad a ordonné l'arrêt des vols internationaux à destination des aéroports du Kurdistan. Pour nous, cela devient une contrainte supplémentaire dans la poursuite de notre enquête, car l'accès au Kurdistan irakien est désormais strictement contrôlé. En contrepartie, les données que nous avons recueillies jusqu'à maintenant prennent une grande valeur, puisqu'il s'agit des données de terrain parmi les plus récentes sur les chrétiens irakiens, dans un contexte où il pourrait s'avérer très complexe pour tout chercheur d'investiguer auprès d'eux dans un avenir rapproché. Qui plus est, les contacts que nous avons noués, et que nous entretenons toujours via les médias sociaux, deviennent plus importants encore afin de demeurer informés de la situation sur place.

Alors que nous aspirions à davantage d'autonomie, peut-être sera-t-il à nouveau préférable pour nous d'envisager de collaborer avec des partenaires sur le terrain si nous souhaitons nous rendre en Irak une autre fois. Les contraintes imposées par les " milieux difficiles » forcent sans cesse le chercheur qui s'y intéresse à s'adapter et à revoir ses plans et ses méthodes. L'Irak, comme exemple, est ainsi un laboratoire 
unique qui oblige à revoir et à développer de nouvelles méthodes d'investigation scientifique.

\section{BIBLIOGRAPHIE}

Althabe, G., Hernandez, V., 2004, « Implication et réflexivité en anthropologie », Journal des anthropologues, 98-99, 15-36.

Benraad, M., 2012, « Fin de l'occupation et crise en Irak : la clé de voûte sunnite », Politique étrangère, 1 (Printemps), 161-172.

Benraad, M., 2015, L'Irak : de Babylone à l'état islamique, Coll. : Idées reçues, Paris, Le Cavalier bleu, $192 \mathrm{p}$.

Boumaza, M., Campana, A., 2007, « Enquêter en milieu difficile », Revue française de sciences politiques, vol. 57, 5-25.

Breaud, S., 1996, «L'usage de l'entretien en sciences sociales. Plaidoyer pour l'entretien ethnographique », Politix, n 35, 226-257.

Claval, P., 1992, « Le thème de la religion dans les études géographiques », Géographie et cultures, no $2,85-110$.

Claval, P., 2012, Géographie culturelle : une nouvelle approche des sociétés et des milieux, Paris, Armand Colin, $2^{\mathrm{e}}$ édition, $351 \mathrm{p}$.

De Charentenay, P., 2008, « À la rencontre des derniers chrétiens d'Irak », Études, 6/408, 809-818.

De Courtois, S., 2009, Le nouveau défi des chrétiens d'Orient. D'Istanbul à Bagdad, Paris, JC Lattès, $232 \mathrm{p}$.

Grawitz, M., 2001, Méthodes des sciences sociales, Paris, Dalloz, 920 p.

Guitton, R., 2011, Ces chrétiens qu'on assassine, Montréal, Flammarion, 311 p.

Houdaille, C., 2017, « Que reproche l'Église de France à l'association SOS Chrétiens d'Orient, La Croix, 9 novembre, [En ligne] : https://www.lacroix.com/Religion/Catholicisme/France/ reproche-lEglise-France-lassociation-SOS-Chretiens-dOrient-2017-11-09-1200890835

Maisterra, P., 2015, « Irak : les chrétiens pris pour cible », Les Cahiers de l'Orient, 1/117, 43-49.

Mellos, K., 2006, « Une science objective ? » in Gauthier, B. (dir), Recherche sociale. De la problématique à la collecte des données, Québec, Presses de l'Université du Québec, 541-559.

Obadia, L., 2007, Anthropologie des religions, Paris, La Découverte, Coll. : Repères, 121 p.

Olivier de Sardan, J.-P., 1995, «La politique du terrain », Enquête, [En ligne] URL : https:// enquete.revues.org/263

Olivier de Sardan, J.-P., 2004 « La rigueur du qualitatif. L'anthropologie comme science empirique », Espace Temps, 84/1, 38-50.

Olivier de Sardan, J.-P., 2008, La rigueur du qualitatif. Les contraintes empiriques de l'interprétation socio-anthropologique, Louvain-la-Neuve, Academia-Bruylant, 368 p. 
Passeron, J.-C., 1991, Le raisonnement sociologique. L'espace non-poppérien du raisonnement naturel, Paris, Nathan, $408 \mathrm{p}$.

Peneff, J., 1990, La méthode biographique : de l'École de Chicago à l'histoire orale, Paris, Armand Colin, $144 \mathrm{p}$.

Poupart, J. (dir.), 2009, La recherche qualitative : enjeux épistémologiques et méthodologiques, Boucherville, Gaetan Morin Éditeur, 405 p.

Roussel, C., 2015, « Nettoyage ethnique, déplacements de population et repeuplement dans le gouvernorat de Ninive (Mossoul, Nord-Irak)», Outre-Terre, 44/3, 250-262.

Sako, L. R., 2015, Ne nous oubliez pas! Le SOS du patriarche des chrétiens d'Irak, Montrouge, Bayard, $151 \mathrm{p}$.

Sleiman, J-B., 2006, Dans le piège irakien : Le cri du cœur de l'archevêque de Bagdad, Paris, Presses de la Renaissance, $185 \mathrm{p}$.

Yacoub, J., 2003, Menaces sur les chrétiens d'Irak, Paris, Éditions CLD, 186 p.

\section{NOTES}

1. Boumaza et Campana se réfèrent à Peneff (1990) qui relate quant à lui les propos que Park tenait à sa classe de sociologie en 1915.

2. Dans les grands centres urbains majoritairement peuplés par des Arabes, les minorités ethniques sont largement arabisées. Il en est de même dans les grandes villes du Kurdistan. Toutefois, dans les zones rurales, les caractéristiques culturelles des minorités ethniques conservent un particularisme important. Par exemple, dans la plaine de Ninive, les chrétiens s'expriment toujours en Soureth, un dérivé de l'Araméen. Les habitants de cette région se définissent également en tant que Syriaques (Assyriens), et non comme des Arabes, bien qu'ils parlent parfaitement l'arabe et qu'ils partagent les us et coutumes de ces-derniers.

3. Les Maadans, beaucoup plus connus sous le nom d'" Arabes des marais ", sont établis de manière traditionnelle dans les marais du sud de l'Irak. De confession chiite, ils ont été arabisés au moment de la conquête arabe du territoire de l'actuel Irak au VII ${ }^{\mathrm{e}}$ siècle.

4. Massoud Barzani a démissionné à l'automne 2017. Le poste est pour le moment toujours vacant.

5. L'appui présumé indéfectible des chrétiens irakiens à Saddam Hussein relèverait davantage de l'obligation que du libre choix (Sleiman, 2006 ; Sako, 2015).

6. Reformulation de propos tenus en anglais ou en arabe, recueillis parmi les déplacés de la plaine de Ninive à l'été 2017.

\section{RÉSUMÉS}

Le contexte qui prévaut dans certains terrains de recherche complique grandement le travail du chercheur. En se basant sur une enquête de terrain réalisée en Irak auprès des déplacés chrétiens de la plaine de Ninive en 2016 et en 2017, cet article propose une réflexion sur les méthodes à adopter afin de garantir la validité d'un processus de collecte de données en « milieu difficile ». 
The context which prevails in certain grounds of research complicates largely the work of the researcher. Based on an experience realized in Iraq in 2016 and 2017, this article suggests a reflection on the methods to adopt to guarantee the validity of a process of research in unfavorable situations.

INDEX

Thèmes : Carnets de terrain

Keywords : Iraqi Christians, Field investigation, Ethnogeography, Methodology

Mots-clés : Chrétiens d'Irak, enquête de terrain, ethnogéographie, méthodologie

\section{AUTEUR}

\section{DAVID VILLENEUVE}

Doctorant

Centre interdisciplinaire de recherche sur l'Afrique et le Moyen-Orient

Université Laval

david.villeneuve.7[at]laval.ca 